\title{
Reducing Road Wear While Ensuring Comfort and Charging Constraints for Dynamically Charged Passenger Vehicles Through Noise-Shaped Path Variations
}

\author{
Clint Ferrin and Randall Christensen, \\ Utah State University, Logan, Utah 84322 USA
}

\begin{abstract}
Dynamically charged vehicles suffer from power loss during wireless power transfer due to receiver and transmitter coil misalignment while driving. Autonomous, dynamically charged vehicles can maximize wireless power transfer by minimizing the misalignment, but the repeated high-precision driving increases road wear. To avoid unnecessary road wear and rutting, a noise shaping filter is proposed that adds variability to a vehicle's trajectory that complies with passenger acceleration and position constraints. However, introducing variability into an optimal charging path also risks depleting battery life prior to destination arrival. Therefore, a path planner is proposed that guarantees average charge within a specified probability and ensures rider comfort while reducing road wear.
\end{abstract}

Index Terms-Shaping filters, Road vehicle control, Wear, Road vehicle power systems.

\section{INTRODUCTION}

$\mathbf{E}$ LECTRIC vehicles (EVs) account for more than $1 \%$ of yearly vehicle sales in the United States, and they are rising in popularity worldwide [1]. Dynamic wireless charging EVs are gaining attention because they decrease weight and cost for EV batteries and eliminate inconvenient charging stops, especially in public transit routes. Lancaster City Park has already produced a fleet of wirelessly charging vehicles that recharge during stops, and four other pilot programs are under development for wireless charging bus systems [2].

Dynamically charging vehicles (DCVs) are vehicles that charge during transit, and they are gaining popularity in public transportation systems because they eliminate overhanging charging wires, improve air quality, and promote quiet transportation. Additionally, charging coils can be installed on public transportation routes to decrease the size, weight, and price of the batteries installed on public transportation fleets, and it is shown to be more practical and cost-effective to install dynamic charging roadways as the number of DCVs increases [3].

One challenge for DCVs is the power loss that occurs due to coil misalignment during wireless power transfer (WPT). Several approaches exist to increase the allowable lateral coil misalignment, but solutions generally include changing the transmitter and receiver coil shapes or adding additional transmitters and coils [4]. These alternatives have the potential to increase both vehicle costs and infrastructure costs. To reduce infrastructure costs, Autonomous Dynamic Charging Vehicles (ADCVs) can be used to achieve lower lateral misalignment errors than humans can achieve, resulting in increased WPT efficiency and decreased infrastructure cost. However, using a vehicle control law that maximizes power transfer with small lateral misalignment has the potential to cause rapid road wear-especially around charging regions.

To avoid excessive road wear, this paper presents a path planner that injects random variation into the path of an autonomous vehicle while ensuring passenger comfort constraints, vehicle position constraints, and a desired WPT charge.

\section{A. Related Research}

1) WPT Lateral Misalignment: Efficiency and maximum power transfer are two important considerations when designing WPT systems, and lateral misalignment can cause a significant reduction to both [5]. Approaches to mitigate the effects of misalignment include changing the number and placement of coils, using shielding methods [6], changing the shape of the coils [7], utilizing orthogonal coil windings [8], and implementing voltage gain controllers [9]. For example, using magnetic-resonance-based WPT with electromagnetic shielding results can extend the permissible lateral misalignment of receiving coils up to $\pm 15 \mathrm{~cm}$ before efficiency degradation and output voltage reduction begins to occur [7], and by increasing the size and number of WPT coils the permissible lateral misalignment tolerance can be increased to tolerances greater than $\pm 40 \mathrm{~cm}$ without significant performance decreases [10].

However, as expressed by Zicheng, there exists a tradeoff between increasing misalignment tolerance and increasing both infrastructure costs and vehicle costs [4]. Therefore, a viable solution to reduce charging coil costs while still ensuring maximum power transfer is to leverage the capabilities of autonomous vehicles to track a precise charging trajectory within centimeters. Unfortunately, such an approach has the potential to wear the road unnecessarily due to the increased vehicle precision, which increases the formation of ruts in the road. Therefore, this research proposes a method to inject maximum variation into a vehicle's trajectory while still obtaining a desired average charging rate, and it assumes a 
misalignment tolerance of $\pm 20 \mathrm{~cm}$, which can realistically be achieved using a voltage gain controller and set of overlapping, laterally displaced pick-up coils [10]. It is also noted that the described methods proposed in this paper are independent of the charging model, and can be applied to any desired charging model.

2) Road Wear: Ruts in the road are caused by densification and shear deformation from repeated vehicle history and weight [11], and they are costly to repair and pose safety concerns due to their effects on hydroplaning [12], [13]. Hjort reports that lateral wander with large variance extends road life-especially with decreasing pavement thickness [14]. Therefore high-precision ADCVs will contribute to the formation of ruts and road wear due to their low lateral variance. Additionally, because ADCVs are primarily implemented on heavier buses for public transportation, they will cause more damage to roads than light-weight consumer vehicles [14]. Therefore, it is desirable that a path planner inject variation into the vehicle's trajectory to prevent unnecessary road wear. For this research, the ratio of permanent strain to temporary strain is used to quantify the amount of road rutting [15].

3) Autonomous Trajectory Tracking: The precision of the sensors used for feedback control determines the ability of autonomous vehicles to track a precisely-defined trajectory. The approaches and corresponding tracking performance vary widely, but two notable approaches with precision sufficient for WPT are magnetic markers and Localizing Ground Penetrating Radar (LGPR). Magnetic markers coupled with odometry have been shown to achieve a mean error of $3 \mathrm{~cm}$ estimation accuracy [16], and LGPR was shown to produce 4.3 $\mathrm{cm}$ estimation accuracy at highway speeds [17]. Such highprecision localization allows for precise trajectory tracking around WPT regions.

Another potential approach for tracking a charging region with low misalignment error is to utilize the instantaneous WPT signal between the receiver and transmitter as a sensor for the control law of the vehicle [18]. Though this technique has not been analyzed in terms of centimeter accuracy, the approach could prove to be beneficial because it does not require additional infrastructure costs to reduce WPT misalignment of a DCV.

Because the sensors outlined in this section can produce high-precision vehicle navigation around WPT charging regions, it is proposed that ADCVs can rely on autonomous navigation for maximizing power transfer on WPT regions that require small lateral misalignment.

4) Passenger Comfort: An important consideration when increasing path variation to reduce road wear is the effect that control variation has on passenger comfort. It is shown in [19] that acceleration discomfort levels for passengers are around $1.2 \mathrm{~m} / \mathrm{s}^{2}$ and acceleration changes of $0.2 \mathrm{~Hz}$ are reported by Golding to increase the likelihood of motion sickness [20]. The duration of exposure to acceleration content also has an effect on motion sickness and can be evaluated using the motion sickness dose value (MSDV), which is accumulated over time [19]. Additionally, the ISO 2631 provides guidance on the combined effect of acceleration, frequency, and duration [21], and is used in many papers to assess the passenger comfort due to vehicle vibrations [22]-[26]. It is further shown by Villagra, that these acceleration constraints can be achieved using a high-level path planner and Clothoids for motion planning [27].

However, intentionally adding path variation to a comfortable trajectory has the potential to induce passenger discomfort due to the added accelerations. Therefore, the design of the induced path variation must be cognizant of these discomfort contributors. To avoid violating acceleration comfort constraints, this paper specifies an upper cutoff frequency of the Power Spectral Density (PSD) to be $3 \mathrm{~dB}$ below the MSDV frequency weighting magnitude to ensure compliance with acceleration frequency constraints. However, because the duration and the corresponding dose of acceleration levels that a passenger is exposed to depend heavily on the design of the charging system, the maximum allowable acceleration and acceleration changes are system dependent.

\section{B. Contributions}

Given the proposed research from Section I-A, the contributions of this work include:

- Generating control-input variations from white Gaussian noise that comply with position and acceleration passenger constraints.

- Calculating the allowable control variation that can be added to a vehicle's charging path while ensuring an average desired WPT charge.

- Ensuring a desired end state of charge when taking into account the vehicle's unique charging profile.

The remainder of the paper begins in Section II by outlining the problem statement and proposing a general form for the noise shaping filter. Next, Section III presents how to select values for the noise shaping filter that comply with passenger position and comfort constraints. Sections IV and V then analyze how to size the output of the noise shaping filter to achieve a desired charge, and Section VI introduces the state-space form of the noise shaping filter. Section VII finally presents simulation results and is followed by concluding remarks.

\section{Problem Statement}

Given a desired reference trajectory, this research aims to develop a noise shaping filter fed by white Gaussian noise that adds variation to a trajectory while complying with the following constraints:

- Position Constraint: Let $\delta$ be the position variation from the center of the lane, then $|\delta| \leq \delta_{\max }$ meters.

- Acceleration Constraint: Let $\alpha$ be the acceleration variation of $\delta$, then $|\alpha| \leq \alpha_{\max } \mathrm{m} / \mathrm{s}^{2}$.

- Acceleration Frequency Constraint: Let $\omega_{\alpha c}$ be the cutoff frequency for $\alpha$, then $\omega_{\alpha c} \leq \omega_{\alpha c \max } \mathrm{rad} / \mathrm{sec}$.

- Average Charge Constraint: Let $q$ be the average WPT charge for an ADCV and let $p$ be a desired probability interval, then $q$ must be achieved within the probability interval $p$ across a charging distance.

To verify the successful compliance of these constraints, Section VII assigns numeric values for each requirement, and 
performs Monte Carlo simulations to verify the successful attainment of each constraint.

\section{A. Proposed Solution}

This section presents the basic form of the noise shaping filter that complies with the constraints outlined in Section II and discusses why the form was adopted. The block diagram for the proposed form with its corresponding power spectral density (PSD) is shown in Figure 1 (a) and (b), where $\alpha$ is the variation in acceleration, $v$ is the variation in velocity, and $\delta$ is the lateral variation from a nominal trajectory, which corresponds to the center of the charging transmitter.

The objective of the noise shaping filter is to bound a 1dimensional point $\delta$ driven by white Gaussian noise without violating the acceleration constraints outlined in Section II, such that the random variable $\delta$ will be added to an ADCV's trajectory to reduce road rutting while ensuring expected charging statistics. It is assumed that the vehicle controller accepts a trajectory with position, velocity, and acceleration, therefore the noise shaping filter must also produce position, velocity, and acceleration outputs. The following discussion addresses why the form for the noise shaping filter depicted in Figure 1 was adopted, and Section III extends the discussion by analyzing how to size the parameters of the noise shaping filter.

To begin the discussion, a heuristic approach to solving the outlined problem is presented that does not comply with the constraints outlined in Section II. The approach is included to aid the reader in understanding restrictions imposed on the noise shaping filter. After it is shown that the heuristic approach will not comply with system constraints, the general form for the noise shaping filter shown in Figure 1 will be presented.

A heuristic approach to bound the random process $\delta$ is to use a second-order Markov driven by white Gaussian noise. The approach seems plausible because a position variance can be set for $\delta$, and the cutoff frequency can be used to bound the frequency changes of $\delta$. Let $\sigma_{\delta}^{2}$ be the variance of $\delta$, then

$$
\sigma_{\delta}^{2}=\frac{1}{2 \pi} \int_{-\infty}^{\infty} \bar{\Psi}_{\delta}(\omega) d \omega,
$$

where $\bar{\Psi}_{\delta}(\omega)$ is the Power Spectral Density (PSD) [28] defined as

$$
\bar{\Psi}_{\delta}(\omega)=\left|G_{\mathrm{lp}}(\omega)\right|^{2} \bar{\Psi}_{n n}(\omega) .
$$

Assuming white Gaussian noise of unity strength and a damping ratio of $\zeta=\frac{1}{\sqrt{2}}$, the PSD of the $\delta$ becomes

$$
\begin{aligned}
\bar{\Psi}_{\delta}(\omega) & =\left|G_{\mathrm{lp}}(\omega)\right|^{2} \bar{\Psi}_{n n}(\omega) \\
\bar{\Psi}_{\delta}(\omega) & =\left|G_{\mathrm{lp}}(\omega)\right|^{2}(1) \\
\left|G_{\mathrm{lp}}(\omega)\right|^{2} & =\frac{\omega_{l}^{4}}{\omega^{4}+\omega_{l}^{4}},
\end{aligned}
$$

as depicted in Figure 2. The variance of position can be bounded using (1) because the integral of (5) is finite, but the corresponding acceleration of $\delta$ cannot be guaranteed to comply with the comfort constraints from Section II. This is because when two derivatives of $\delta$ are taken to find $\alpha$, the resulting system magnitude squared becomes

$$
\bar{\Psi}_{\alpha}(\omega)=\frac{\omega_{l}^{4} \omega^{4}}{\omega^{4}+\omega_{l}^{4}},
$$

and the corresponding variance of the acceleration becomes

$$
\sigma_{\alpha}^{2}=\frac{1}{2 \pi} \int_{-\infty}^{\infty} \bar{\Psi}_{\alpha}(\omega) d \omega=\infty,
$$

which implies that the variance of acceleration is infinite, and cannot be statistically bounded as seen in Figure 2 .

To overcome the problem of unbounded acceleration variations, a higher-order filter can be utilized that induces a faster roll-off rate, which in turn makes $\sigma_{\alpha}^{2}$ finite. It is also proposed that the variation for $\alpha$ be shaped before $\delta$, then integrated twice. This ordering is desirable because the initial shaping of $\alpha$ facilitates the placement of the upper cutoff frequency for the acceleration constraint outlined in Section II. Therefore, consider a band-pass filter composed of a secondorder low-pass filter and a second-order high-pass filter derived in Appendix A. The resulting PSD is

$$
|G(\omega)|^{2}=\frac{K \omega_{l}^{4} \omega^{4}}{\left(\omega^{4}+\omega_{l}^{4}\right)\left(\omega^{4}+\omega_{h}^{4}\right)}
$$

where $\omega_{l}$ is the cut-off frequency for the low-pass filter, $\omega_{h}$ is the cut-off frequency for the high-pass filter, and $K$ is the system gain. Let $\delta_{\alpha}^{2}$ be the variance $\alpha$, then as shown in Figure 1 (b), an upper bound can be put on $\delta_{\alpha}^{2}$ because the integral of $|G(\omega)|^{2}$ is finite. Due to the higher-order system, when $G(s)$ is integrated twice to find $\delta$, the integral of the PSD for $\delta$ is also finite, which allows bounds to be placed on the position variance as well. Therefore, the architecture outlined in Figure 1 (a) can be used to shape white Gaussian noise and comply with all of the constraints by carefully selecting values for $\omega_{l}$, $\omega_{h}$, and $K$, as outlined in Section II.

As a result, (9), (10), and (11) characterize the general relationship of the comfort constraints to the noise shaping filter where $\rho$ is the desired magnitude attenuation at the frequency $\omega_{\alpha c \max }$ in $\mathrm{rad} / \mathrm{sec}$.

$$
\begin{aligned}
\sigma_{\alpha}^{2} & =\frac{1}{2 \pi} \int_{-\infty}^{\infty}|G(\omega)|^{2} d \omega \\
\sigma_{\delta}^{2} & =\frac{1}{2 \pi} \int_{-\infty}^{\infty}|H(\omega)|^{2} d \omega \\
\rho & =\left|G\left(\omega_{\alpha c \max }\right)\right|^{2}
\end{aligned}
$$

Equation (9) is used to bound the acceleration of the system, (10) is used to bound the distance of the vehicle from the center of the trajectory, and (11) is used to set the magnitude of the upper cutoff frequency at $\omega_{\alpha c \max }$ to the desired attenuation.

\section{B. Performance Evaluation}

This section outlines how the reduction in road wear is measured in terms of strain. The plastic, or permanent, strain $\left(\epsilon_{p}\right)$ on pavement is a function of temperature $(T)$, the number of cycles $(N)$, and the traffic-induced vertical strain $\left(\epsilon_{z z}\right)$, 


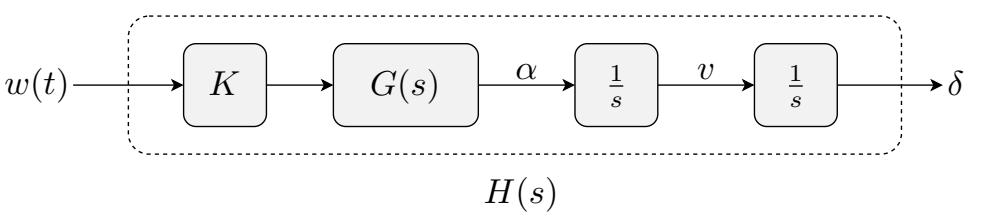

(a)

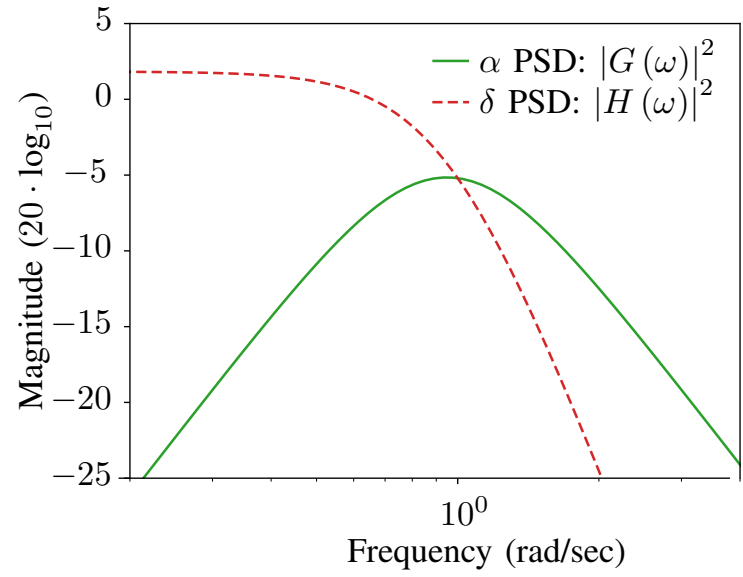

(b)

Figure 1: (a) Block diagram for the noise shaping filter $H(s)$, where $\omega(t), \alpha, v$, and $\delta$ are white Gaussian noise, acceleration variation, velocity variation, and position variation respectively. (b) Power spectral density of the band-pass filter $G(s)$ and $H(s)$ used to produce acceleration variation and position variation respectively. Both PSDs are finite, which implies the noise shaping filter can be designed to comply with acceleration and position comfort constraints.

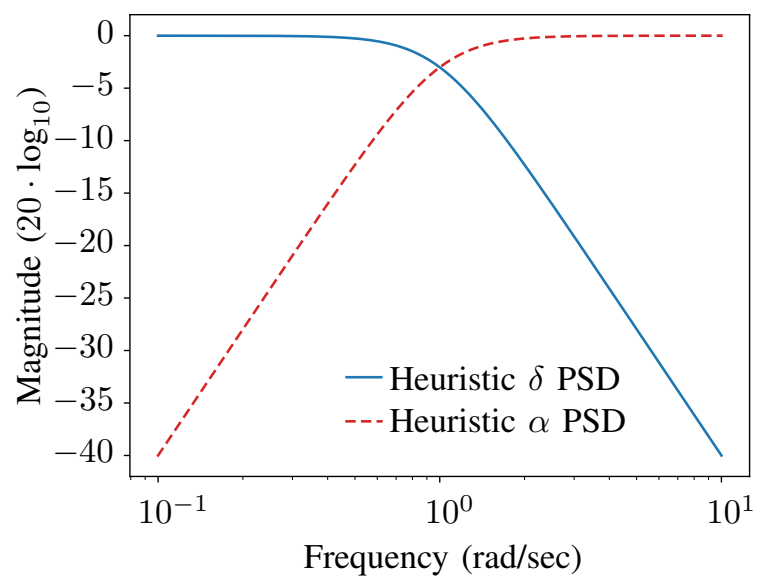

Figure 2: A heuristic approach to bounding position variation with a second-order low-pass filter. Though the integral of the Power Spectral Density of $\delta$ is finite, the double integral of the PSD for $\alpha$ is infinite.

and is often expressed as the plastic strain normalized by the traffic-induced strain [15].

$$
\frac{\epsilon_{p}}{\epsilon_{z z}}=k_{1} \times 10^{k_{r 1}} T^{1.5606} N^{0.4791}
$$

The calibration constants $k_{1}$, and $k_{r 1}$ used in this research are representative of the strain of unmodified asphalt concrete with a thickness of $h_{a c}=10.2 \mathrm{~cm}$ evaluated at a depth of $1.27 \mathrm{~cm}$ [29]

$$
\begin{gathered}
k_{r 1}=-3.35412 \\
k_{1}=\left(C_{1}+C_{2} \cdot \operatorname{depth}\right) \cdot 0.328196^{\text {depth }}
\end{gathered}
$$

where

$$
\begin{gathered}
C_{1}=-0.1039 \cdot h_{a c}^{2}+2.4868 \cdot h_{a c}-17.342 \\
C_{2}=0.0172 \cdot h_{a c}^{2}-1.7331 \cdot h_{a c}+27.428
\end{gathered}
$$

The temperature is chosen to be $T=21 C$ and the total number of cycles $N=10,000$.

In the case of random lateral wander, the number of cycles experienced by a section of road and the resulting strain is a random variable. Expressing (12) for a given lateral wander interval $d_{i}$ yields the strain experienced by the interval.

$$
\frac{\epsilon_{p}\left(d_{i}\right)}{\epsilon_{z z}}=k_{1} \times 10^{k_{r 1}} T^{1.5606} N\left(d_{i}\right)^{0.4791}
$$

where the number of cycles in the interval $N\left(d_{i}\right)$ is obtained from the histogram of lateral wander with an assumed number of vehicle passes. Equation (17) provides a histogram of normalized plastic strain vs. lateral wander, whose maximum is used to assess the degree to which the proposed algorithm reduces road wear. The process for performance evaluation is illustrated in Figure 3. The histogram in Figure 3 (a) shows the number of passes for two lateral wander levels of $25 \mathrm{~cm}$ and $75 \mathrm{~cm}$. For illustration purposes, the width of the interval and number of vehicles is arbitrarily chosen to be $5 \mathrm{~cm}$ and 10,000 vehicles. The histogram in Figure 3 (b) shows the normalized strain as calculated from (17) for each $5 \mathrm{~cm}$ interval. The increased lateral wander results in a reduction in the peak of the normalized strain histogram (i.e. maximum normalized strain) and a more even road wear when compared to the lower lateral wander. For this research, the reduction in road wear is quantified as the reduction in the maximum normalized strain, which corresponds to a factor of 0.6 for this example. For the results presented in Section VIII, histograms are analyzed at various points along the road. The performance is therefore illustrated by the maximum normalized strain as a function of path length.

\section{Shaping Filter Parameter Selection}

This section analyzes how to select values for the noise shaping filter parameters $\omega_{l}, \omega_{h}$, and $K$ that comply with the position and comfort criteria outlined in Section II. First, three equations are presented that relate the system parameters 


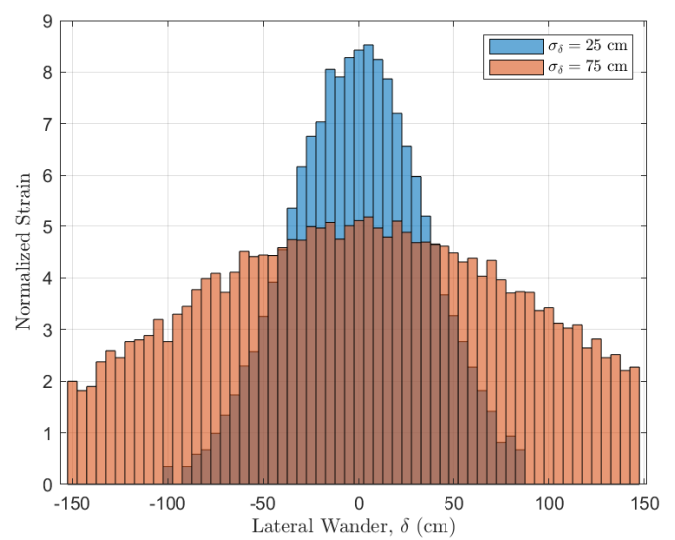

(a)

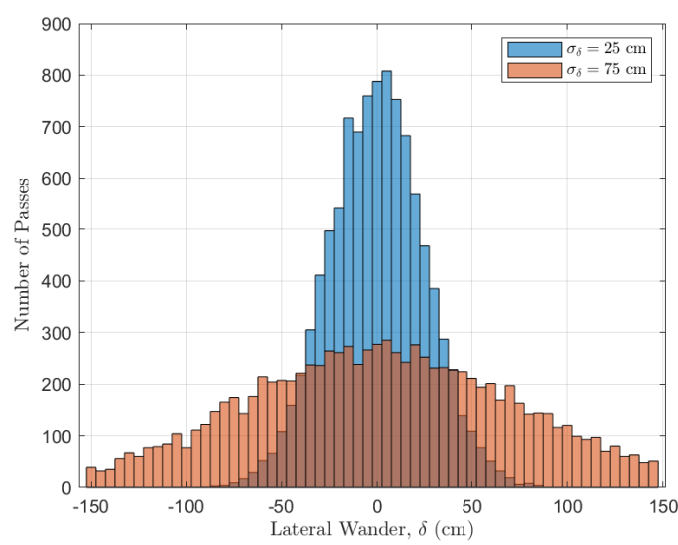

(b)

Figure 3: Illustration of performance evaluation. (a) Histogram of the number of vehicle passes. (b) The resulting normalized strain for a given lateral wander interval.

to the desired constraints. Next, Section III-A sets the values for $\omega_{l}$ and $\omega_{h}$ using the maximum desired acceleration cutoff frequency. Finally, Section III-B proposes how to size $K$ based on a desired position variance.

Using the general form of the filter proposed in Section II-A, the three equations that relate the system parameters to the desired constraints are

$$
\begin{aligned}
\sigma_{\alpha}^{2} & =\frac{1}{2 \pi} \int_{-\infty}^{\infty} \frac{K^{2} \omega_{l}^{4} \omega^{4}}{\left(\omega^{4}+\omega_{l}^{4}\right)\left(\omega^{4}+\omega_{h}^{4}\right)} d \omega \\
\sigma_{\delta}^{2} & =\frac{1}{2 \pi} \int_{-\infty}^{\infty} \frac{K^{2} \omega_{l}^{4}}{\left(\omega^{4}+\omega_{l}^{4}\right)\left(\omega^{4}+\omega_{h}^{4}\right)} d \omega \\
\rho & =\frac{K \omega_{l}^{2} \omega_{\alpha c}^{2}}{\left(\omega_{\alpha c}^{4}+\omega_{l}^{4}\right)\left(\omega_{\alpha c}^{4}+\omega_{h}^{4}\right)},
\end{aligned}
$$

where $\omega_{\alpha c}$ is the maximum upper cutoff frequency to achieve acceleration frequency constraints in $\mathrm{rad} / \mathrm{sec}$, and $\rho$ is the desired magnitude attenuation at the frequency $\omega_{\alpha c}$.

It is proposed that these equations be used to define the noise shaping filter because the three constraints are governed by the three design variables $\omega_{l}, \omega_{h}$, and $K$. One approach to solve for $\omega_{l}, \omega_{h}$, and $K$ is to calculate the indefinite integrals of $\sigma_{\alpha}^{2}$ and $\sigma_{\delta}^{2}$, then to algebraically relate the equations using three equations and three unknowns. However, this approach proves to be non-trivial due to the eighth order polynomial in the denominator of the integrand of (18) and (19). An alternative solution that solves for optimal values of $\omega_{l}, \omega_{h}$, and $K$ is to first select permissible values of $\omega_{l}$ and $\omega_{h}$ which satisfy the cutoff frequency $\omega_{\alpha c \max }$, then scale the incoming noise by $K$ to meet the other system constraints, as will be shown in Section III-B. This approach allows the designer to adjust the values of $\omega_{l}$ and $\omega_{h}$ to tune the overall cutoff frequency and the maximum position variance based on the system application.

\section{A. Selecting $\omega_{l}$ and $\omega_{h}$}

This section presents how to select values for $\omega_{l}$ and $\omega_{h}$ based on the desired acceleration profile and cutoff frequency.
To begin the discussion on choosing values for $\omega_{l}$ and $\omega_{h}$, only the case when

$$
\omega_{h}=\kappa \omega_{l}
$$

such that $\kappa \geq 1$ is considered, where $\kappa$ is used to scale the width of the band-pass filter. One property of increasing $\kappa$, is that as $\kappa$ increases the cutoff frequency for $|H(\omega)|^{2}$ decreases, which corresponds to a decreased position variation as observed in Figure 4.

The value of $\kappa$ is, therefore, a tuning parameter that is governed by the passenger comfort requirements. The upper cutoff frequency of $|G(\omega)|^{2}$ is limited by the maximum desired acceleration cutoff frequency, but $\kappa$ can be increased or decreased based on system design. However, there exists a trade-off between the width of the pass-band filter $|G(\omega)|^{2}$ and the cutoff frequency of $|H(\omega)|^{2}$; a narrower pass-band results in a noise pattern that tends towards oscillatory accelerations with a frequency of $\omega_{l}$. For this system, a higher cutoff frequency is beneficial because it increases the likelihood of crossing the center of the charging pad, enabling a more consistent average charge. This reduces the likelihood that a vehicle drives outside the maximum charge region at a constant offset for an extended period of time. In contrast, a small lower cuttoff frequency, $\omega_{l}$, increases the pass-band width, resulting in more low-frequency content of the path variation, and higher likelihood of traveling outside the charging region. Therefore, the results in this work assume that $\kappa=1$.

With the pass-band width set by $\kappa$, the selection of $\omega_{l}$ will now be analyzed. The parameter $\omega_{l}$ must be selected such that the magnitude of the upper cutoff frequency of $|G(\omega)|^{2}$ has a desired attenuation of $\rho$. Given a desired $\kappa, \omega_{a c}$, and $\rho$, the value for $\omega_{l}$ as derived in Appendix B is

$$
\omega_{l}=\left(-\sqrt{-\frac{\omega_{\alpha c}^{8}}{\kappa^{4}}+\frac{1}{4} \Omega^{2}}-\frac{1}{2} \Omega\right)^{1 / 4},
$$




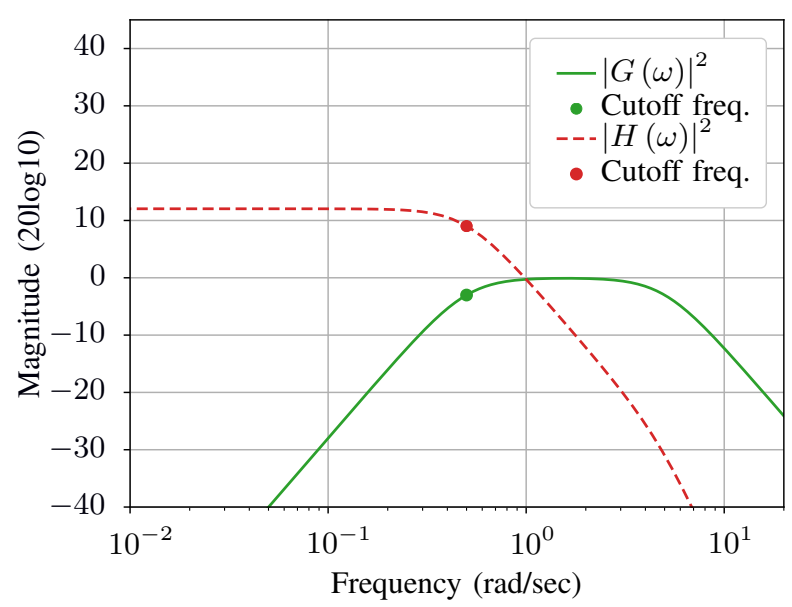

(a)

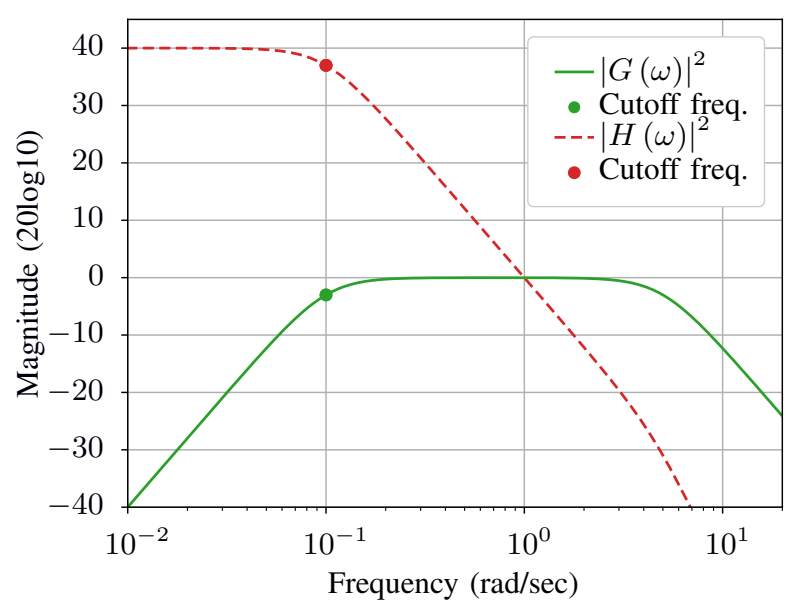

(b)

Figure 4: Plots demonstrating that the width of the band-pass filter set by $\kappa$ determines the cut-off frequency of the position variance. (a) Frequency domain relationship between a small value of $\kappa$ and a higher cut-off frequency of the position variance. (b) Frequency domain relationship between a large value of $\kappa$ and a lower cut-off frequency of the position variance.

where

$$
\Omega=\omega_{\alpha c}^{4}\left(1+\frac{1}{\kappa^{4}}-\frac{\left(\kappa^{2}+1\right)^{2}}{\kappa^{4} \rho^{2}}\right)
$$

and $\omega_{h}$ is trivially solved for using (21). The selection of $\omega_{a c}$ and $\rho$ are left to the system designer given knowledge of the expected motion sickness dose value for the charge station frequency and length.

\section{B. Calculating $K$ from Desired Position Variance}

As discussed in Section II-A, the integral values for $\sigma_{\alpha}^{2}$ and $\sigma_{\delta}^{2}$ are difficult to relate when trying to simultaneously solve for $K, \omega_{l}$, and $\omega_{h}$. However, with $\omega_{l}$ and $\omega_{h}$ fixed using the method from the previous section, the integral terms of (18) and (19) can be computed numerically, so that $\sigma_{\alpha}^{2}$ and $\sigma_{\delta}^{2}$ become quadratic functions of $K$ scaled by a constant. Let the numeric solution to the scaled integral terms of (18) and (19) be represented as $\gamma_{\alpha}$, and $\gamma_{\delta}$ respectively, then

$$
\begin{aligned}
& \sigma_{\alpha}^{2}=\gamma_{\alpha} K^{2} \\
& \sigma_{\delta}^{2}=\gamma_{\delta} K^{2} .
\end{aligned}
$$

Using (25), $K$ can be selected to achieve a desired position variation using

$$
K=\sqrt{\sigma_{\delta \text { desired }}^{2} / \gamma_{\delta}}
$$

However, the maximum value of $K$ is constrained by both $\alpha_{\max }$ and $\delta_{\max }$, therefore

$$
K_{\max }=\min \left(\sqrt{\sigma_{\alpha \max }^{2} / \gamma_{\alpha}}, \sqrt{\sigma_{\delta \max }^{2} / \gamma_{\delta}}\right) .
$$

Thus, depending on the values of the position and acceleration requirements, the maximum position variation may not be achieved. For cases where maximum position variation is important, the width of the pass-band can be increased to allow for larger values of $\sigma_{\delta}^{2}$. However, as explained in Section III-A, increasing the width of the pass-band comes at the cost of reducing the cutoff frequency for position changes governed by $|H(\omega)|^{2}$. Therefore (26) can be used in conjunction with (27) to set a bound on position variation while complying with comfort constraints from Section II.

However, it is important to note that the design of the noise shaping filter can statistically bound the probability that the position variation violates the $\delta_{\max }$ boundary, but the probability that such a violation will occur is greater than zero, albeit small by design. Consequently, for practical implementation, additional precautions should be taken to eliminate the possibility that the control variation sends a vehicle beyond $\delta_{\max }$. This can be achieved by clamping the output of $G(s)$ to only accelerate towards the center of the lane when the vehicle is near the $\delta_{\max }$ boundary.

\section{Calculating $K$ From Desired Average Charge}

Section III-B analyzes how to select $K$ to achieve a desired position variation within comfort constraints, but it does not address how the variations affect dynamic charging. This section extends Section III-B by sizing $K$ to achieve a desired average charge based on non-linear WPT charging equations within a probability interval. The section begins by defining the model used for simulating the WPT charging equations, and it proposes a method to predict average charge as a function of $K$.

Assuming a coil arrangement proposed by Choi [10], WPT charging experiences a full charge region towards the center of the charging pads, and a charge transition region as lateral misalignment increases as seen in Figure 5 (a). The nonlinear charging equations used to model charge degradation based on lateral misalignment can then be approximated as a two-sided Sigmoid function. The piecewise equations used for calculating charge based on lateral misalignment are

$$
f(\delta)=\left\{\begin{array}{cc}
Q_{\max } & |\delta| \leq R_{f} / 2 \\
\frac{Q_{\max }}{1+e^{w(x-c)}} & R_{f} / 2<|\delta|<R_{t} \\
0 & |\delta| \geq R_{f} / 2+R_{t}
\end{array},\right.
$$




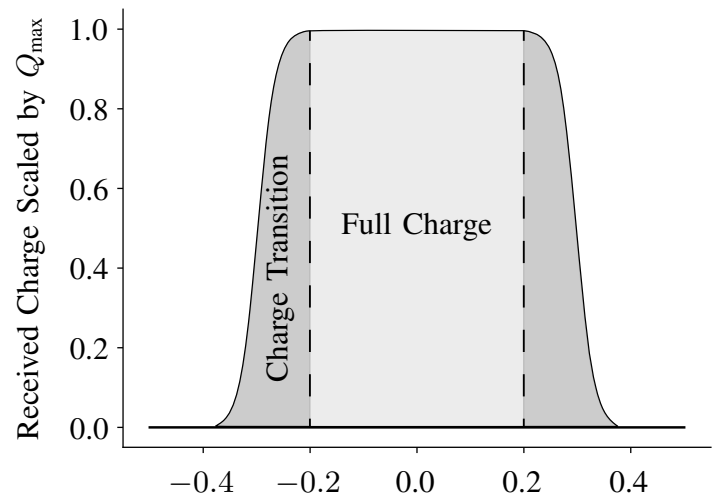

Lateral Misalignment, $\delta$, from Center of Transmitter $(m)$

(a)

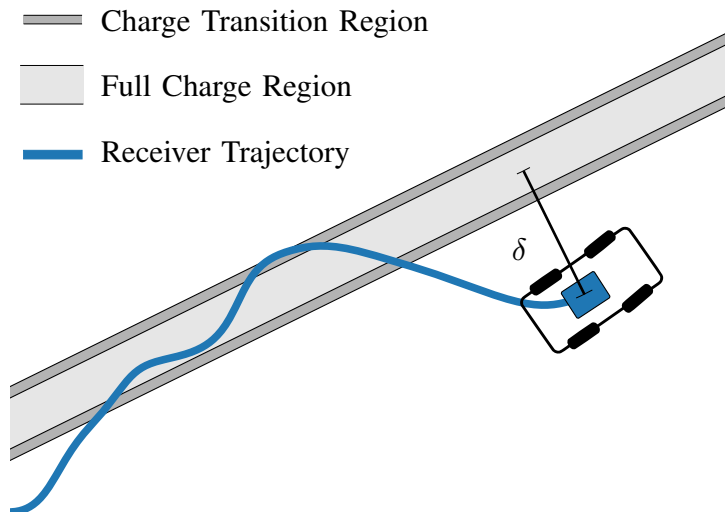

(b)

Figure 5: (a) Unscaled charging function relating distance from the center of the charging coil to the received WPT charge with Full Charge Region width of $R_{f}=0.4 m$ and the Charge Transition Region width of $R_{t}=0.2 m$. (b) Illustration of lateral misalignment of the receiver from the WPT transmitter, represented by $\delta$.

where $\delta$ is lateral misalignment, $Q_{\max }$ is the maximum charge delivered, $R_{f}$ is the width of the full charge region, $R_{t}$ is the width of the charge transition region, $w=12 / R_{t}$, and $c=R_{f} / 2+R_{t} / 2$. A pictorial representation of how (28) relates to the overall problem can be seen in Figure 5 (b), where the trajectory of the WPT receiver is depicted. As the position of the WPT receiver in Figure 5 (b) varies, the lane will be driven more evenly, but the lateral distance of the WPT receiver will also vary, based on (28). It should also be noted that because (28) is non-linear, a sample-based statistical method is employed to formulate a function that returns the average charge based on the induced path variation caused by the size of $K$.

The metric used to determine how variations in $\delta$ affect WPT over a charging region is the average charge, which can be expressed as

$$
q_{\text {avg }}=\frac{1}{t_{f}-t_{0}} \int_{t_{0}}^{t_{f}} q(t) d t
$$

where $q(t)$ is the instantaneous charge received at time $t, t_{0}$ is the initial time, and $t_{f}$ is the final time of the analysis. Because $q(t)$ is a random variable, $q_{\text {avg }}$ is also a random variable and Monte Carlo analysis can be applied to estimate its mean and standard deviation, denoted as $\mu_{q}$ and $\sigma_{q}$ respectively. To relate how induced path variation caused by $K$ affects $q_{\text {avg }}$, both $\mu_{q}$ and $\sigma_{q}$ are calculated for incremental values of $K$ on a straight charging path-assuming the simulation parameters outlined in Table I, where the explanation of the parameter selection is addressed in Section VII. The calculated results for $\mu_{q}$ and $\sigma_{q}$ at increasing values of $K$ can be seen in Figure 6 (a) and (b) respectively with corresponding confidence intervals calculated from the Monte Carlo simulations.

As seen in Figure 6, both $\mu_{q}$ and $\sigma_{q}$ can be approximated as a function of $K$ using second-order polynomials. This approximation is useful because it provides equations that can be used to calculate the average charge and confidence interval without storing or re-running the Monte Carlo simulation. The
Table I: Parameter list for Monte Carlo simulations.

\begin{tabular}{ll}
\hline Parameter & Value \\
\hline$\omega_{l}$ - Low-pass filter cutoff frequency & $0.809 \mathrm{rad} / \mathrm{s}$ \\
$\omega_{h}$ - High-pass filter cutoff frequency & $0.809 \mathrm{rad} / \mathrm{s}$ \\
$Q_{\max }$ - Maximum Charge Delivery & $60 \mathrm{kilowatts}$ \\
$R_{f}$ - Width of Full Charge Region & $0.40 \mathrm{~m}$ \\
$R_{t}$ - Width of Charge Transition Region & $0.20 \mathrm{~m}$ \\
$\delta_{\max }$ - Maximum Lateral Misalignment & $0.88 \mathrm{~m}$ \\
$\alpha_{\max }$ - Maximum Acceleration & $1.2 \mathrm{~m} / \mathrm{s}^{2}$ \\
$\omega_{\alpha c \max }$ - Maximum Acceleration Changes & $0.2 \mathrm{~Hz}$ \\
$\rho$ - Magnitude Attenuation at $\omega_{\alpha c \max }$ & $0.2 \mathrm{~Hz}$ \\
Analysis Start Time & $0 \mathrm{~s}$ \\
Monte Carlo Simulations & 250 \\
Trajectory Length & $200 \mathrm{~m}$ \\
Trajectory Velocity & $5 \mathrm{~m} / \mathrm{s}$ \\
\hline
\end{tabular}

polynomial approximation for the expected average charge becomes

$$
\begin{aligned}
& \hat{\mu}_{q}=a_{\mu} K^{2}+b_{\mu} K+c_{\mu} \\
& \hat{\mu}_{q}=10796.0 K^{2}-55177.8 K+70945.6,
\end{aligned}
$$

and the polynomial approximation for the standard deviation of average charge is

$$
\begin{aligned}
& \hat{\sigma}_{q}=a_{\sigma} K^{2}+b_{\sigma} K+c_{\sigma} \\
& \hat{\sigma}_{q}=-24195.9 K^{2}+36538.7 K-5055.5
\end{aligned}
$$

Additionally, (32) can be extended to represent any standard deviation away from the mean by multiplying (32) by $z$, where $z$ is the number of desired standard deviations. Therefore

$$
\hat{\sigma}_{q}^{\prime}=z\left(a_{\sigma} K^{2}+b_{\sigma} K+c_{\sigma}\right) .
$$

The standard deviation can then be used to specify the likelihood of achieving a desired average charge $q_{d}$ with probability $p$, where $p$ is the lower-tail integral of $z$. The function that returns expected charge $q_{d}$ given a desired probability based on $z$ becomes

$$
\begin{aligned}
& q_{d}=q_{\mathrm{avg}}-\hat{\sigma}_{q}^{\prime} \\
& q_{d}=\left(a_{\mu}-z a_{\sigma}\right) K^{2}+\left(b_{\mu}-z b_{\sigma}\right) K+\left(c_{\mu}-z c_{\sigma}\right)
\end{aligned}
$$




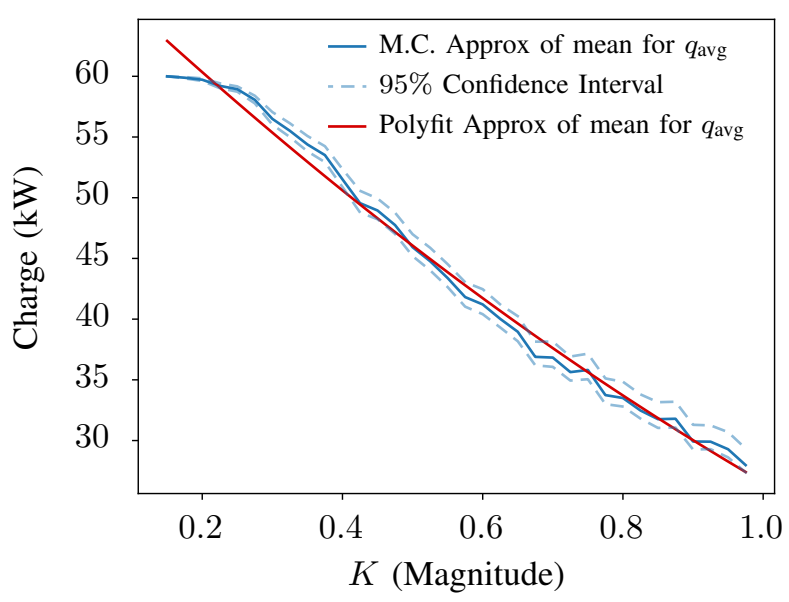

(a)

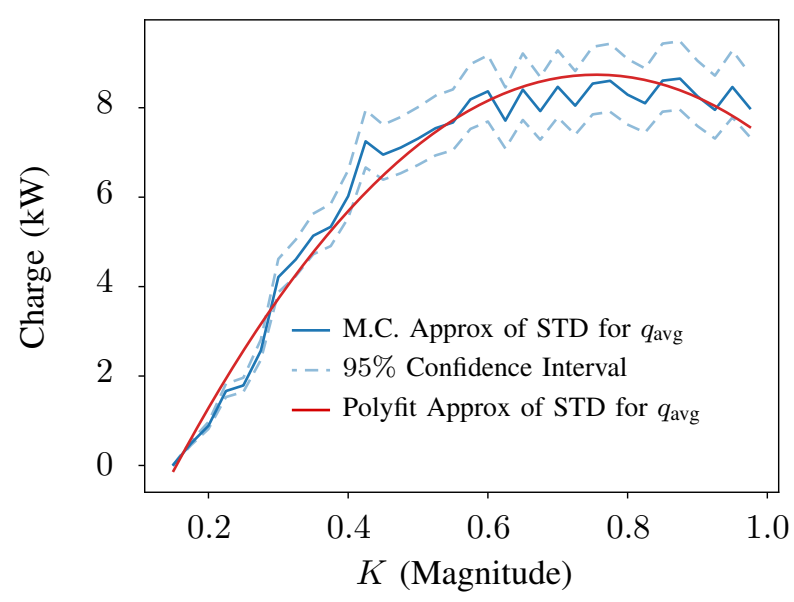

(b)

Figure 6: Nonlinear approximation of charge and standard deviation fit with second-order polynomials taken from a Monte Carlo Simulation of 250 runs on a straight charging path of 200 meters. (a) Approximation for the mean of the average charge $q_{\text {avg }}$ as a function of $K$ fit with a second-order polynomial with a 95\% confidence interval. (b) Approximation for the standard deviation of the average charge $q_{\text {avg }}$ as a function of $K$ fit with a second-order polynomial with a $95 \%$ confidence interval.

and the corresponding $K$ value to achieve $q_{d}$ is solved for in (35) by completing the square, and is written as

$$
\begin{aligned}
& K= \\
& -\sqrt{\frac{q_{d}-\left(c_{\mu}-z c_{\sigma}\right)}{\left(a_{\mu}-z a_{\sigma}\right)}+\frac{1}{4} \frac{\left(b_{\mu}-z b_{\sigma}\right)^{2}}{\left(a_{\mu}-z a_{\sigma}\right)^{2}}}-\frac{1}{2} \frac{\left(b_{\mu}-z b_{\sigma}\right)}{\left(a_{\mu}-z a_{\sigma}\right)}
\end{aligned}
$$

It should be noted that this method is computationally efficient because using (35) to calculate $K$ allows a vehicle's path planner to make charge estimates by solving a single equation. Therefore, the Monte Carlo simulations need only be run during the initial analysis and referenced during vehicle path planning.

\section{Calculating $K$ From Desired Change in State OF CHARGE}

The previous section presents a method for scaling the noise shaping filter output to achieve a desired average charge. This section presents a method for sizing the output of the noise shaping filter to achieve a desired change in the state of charge (SOC) within a fixed distance and within a probability interval. However, to ensure a desired change in SOC in a fixed distance, the estimated velocity and rate of battery discharge unique to the vehicle's driving profile must be considered. Therefore, it is the responsibility of the vehicle's path planner to estimate a desired average charge based on the vehicle's unique driving profile. For simulation purposes, this paper assumes the discharge of the battery is based on the Newtonian equations of motion where consumed power is equal to the sum of all of the forces acting on the vehicle multiplied by velocity [30].

One approach to estimating the desired change in SOC is to subtract the estimated average power consumption $r_{\text {avg }}$ of the battery from the average charge $q_{\text {avg }}$ over their respective time periods. Let $\Delta Q$ be the change of SOC in watt-hours from the current time $t$ to the time it takes to arrive at the desired destination, then

$$
\Delta Q=q_{\mathrm{avg}} t_{q}-r_{\mathrm{avg}} t_{r}
$$

where $t_{q}$ is the amount of time the battery is receiving power, and $t_{r}$ is the amount of time the battery is using power. It then follows that the desired average charge to achieve a desired $\Delta Q$ is

$$
q_{d}=\left(\Delta Q_{\mathrm{wH}}+r_{\mathrm{avg}} t_{r}\right) / t_{q} .
$$

Therefore, to ensure a desired $\Delta Q$ at a final destination, the variables $t_{r}, t_{q}$, and $r_{\text {avg }}$ must be estimated. The time required to reach the destination can be estimated as

$$
t_{r}=\frac{d}{v}
$$

where $d$ is the total distance to the destination, and the velocity $v$ is assumed to be constant. The time the vehicle receives charge can be estimated

$$
t_{q}=\frac{d_{q}}{v}
$$

where $d_{q}$ is the lengths of all charging regions between the current position and the destination, and $v$ is defined as in (39). As for estimating $r_{\mathrm{avg}}$, it is necessary to estimate the battery SOC to analyze the change of charge. Because SOC estimation is a complex problem being addressed by many researchers [31], [32], this research assumes the path planner has perfect knowledge of the battery SOC, therefore $r_{\text {avg }}$ can be estimated based on the average battery discharge over a time period. An estimate for $r_{\text {avg }}$ can be obtained as

$$
r_{\mathrm{avg}}=\frac{1}{t_{a}} \Delta Q_{a}
$$

where $t_{a}$ is the amount of time over which to average a change in SOC, and $\Delta Q_{a}$ is the change of SOC in watt-hours from $t$ to a previous time $t-t_{a}$. 
Because $t_{r}, t_{q}$, and $r_{\text {avg }}$ can be estimated using (39), (40) and (41) respectively, the necessary value of $q_{d}$ to achieve a desired $\Delta Q$ can be calculated using (38). With a desired $q_{d}$ set, the sizing for $K$ can then be determined according to the analysis presented in Section IV, and the corresponding results are presented in Section VII-C.

\section{Noise Shaping Filter IMPLEMENTATion}

This section addresses how to generate the output of the noise shaping filter and implement it with a vehicle's control law. The noise shaping filter can be put into standard canonical form in the Laplace domain to produce the state-space dynamic equations. The Laplace representation of the bandpass filter generated from a second-order low-pass filter and a second-order high-pass filter is

$$
G(s)=\frac{K b s^{2}}{s^{4}+a_{1} s^{3}+a_{2} s^{2}+a_{3} s+a_{4}}
$$

where

$$
\begin{aligned}
a_{1} & =2 \omega_{h} \zeta+2 \omega_{l} \zeta \\
a_{2} & =\omega_{h}^{2}+4 \omega_{h} \omega_{l} \zeta^{2}+\omega_{l}^{2} \\
a_{3} & =2 \omega_{h}^{2} \omega_{l}+2 \omega_{h} \omega_{l}^{2} \\
a_{4} & =\omega_{h}^{2} \omega_{l}^{2} \\
b & =\omega_{l}^{2} .
\end{aligned}
$$

Equation (42) can then be put into the Controllable Canonical Form such that

$$
\begin{aligned}
& \mathbf{A}=\left[\begin{array}{cccc}
0 & 1 & 0 & 0 \\
0 & 0 & 1 & 0 \\
0 & 0 & 0 & 1 \\
-a_{4} & -a_{3} & -a_{2} & -a_{1}
\end{array}\right] \\
& \mathbf{B}=\left[\begin{array}{c}
0 \\
0 \\
0 \\
1
\end{array}\right] \\
& \mathbf{C}=\left[\begin{array}{cccc}
0 & 0 & K b & 0 \\
0 & K b & 0 & 0 \\
K b & 0 & 0 & 0
\end{array}\right] \\
& \mathbf{D}=\mathbf{0}
\end{aligned}
$$

Equations (44) through (47) form the state space dynamic equations required to produce variations in acceleration $\alpha$, velocity $v$, and position $\delta$. Therefore, the variation system becomes

$$
\underline{\dot{x}}=\mathbf{A} \underline{x}+\mathbf{B} w(t)
$$

which produces the output

$$
\left[\begin{array}{l}
\alpha \\
v \\
\delta
\end{array}\right]=\mathbf{C} \underline{\mathbf{x}} .
$$

With the acceleration, velocity, and position outputs generated, the trajectory states can then be added to the vehicle's trajectory, as seen in Figure 7. It is also significant that the noise shaping filter algorithm is controller agnostic because the trajectory variations are directly added to the desired trajectory, as shown in Figure 7 . This allows the noise shaping filter algorithm to be applied in a variety of to existing vehicle models and in a variety of settings.

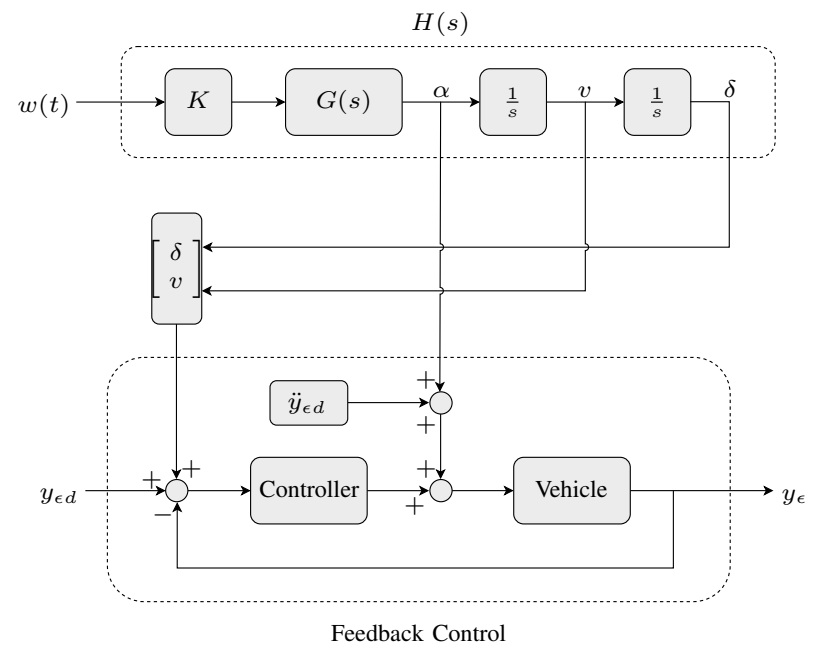

Figure 7: Block diagram depicting how the noise shaping filter output is directly incorporated into the vehicle path through its control inputs.

\section{Simulation Results for Desired AVERAGE CHARGE}

This section verifies the compliance with system constraints outlined in Section II and gives a brief analysis of the results. It outlines the system models used during simulation, it explains the goal of each simulation, and it reports on the results of the simulations.

The vehicle model used for simulation was the Ford Focus 2013 EV with bicycle kinematic equations [27], and the battery model used to determine SOC was the Newtonian propulsion model for battery discharge [30] and a set of overlapping, laterally displaced pick-up coils to model battery charge [10]. The system dynamics can therefore succinctly be modeled as

$$
\left[\begin{array}{c}
\dot{x} \\
\dot{y} \\
\dot{\psi} \\
Q \\
\dot{v} \\
\dot{\phi}
\end{array}\right]=\left[\begin{array}{c}
v \cdot \cos \psi \\
v \cdot \sin \psi \\
\frac{v}{L} \tan \phi \\
q-r \\
0 \\
0
\end{array}\right]+\left[\begin{array}{ll}
0 & 0 \\
0 & 0 \\
0 & 0 \\
0 & 0 \\
1 & 0 \\
0 & 1
\end{array}\right]\left[\begin{array}{l}
a \\
\xi
\end{array}\right]
$$

where $(x, y)$ is the position of the vehicle, $\psi$ is the heading, $\mathrm{Q}$ is the SOC, $v$ is the translational velocity, $\phi$ is the steering angle, $L$ is the vehicle wheelbase, $q$ is the instantaneous battery power received using (28), and $r$ is the instantaneous battery power depleted based on resistive forces [30]. The inputs to the system are $a$ and $\xi$ which are the longitudinal acceleration and change in steering angle respectively.

The goal of the simulations in this section is to verify that the vehicle achieved an average charge of 40 kilowatts with a probability of 0.97 based on 250 Monte Carlo simulations, while injecting maximum variation into the trajectory and complying with system constraints from Section II. Using (36), the calculated maximum value for $K$ was 0.38 , and each test 
assumed the simulation properties in Table I unless otherwise specified. It should also be noted that these simulations focus on the effects that vehicle input variations have on passenger comfort, so disturbances caused by vehicle vibrations or vehicle suspension are not considered, and the parameters chosen for illustration of the algorithm are not generic. It is the responsibility of the system designer to select values parameter values given knowledge of the system which is comprised of the frequency and duration of charging stations. For these simulations, $\omega_{\alpha c}$ and $\rho$ were chosen to achieve a $-3 \mathrm{~dB}$ attenuation for the MSDV frequency weighting function.

\section{A. Results for Acceleration Comfort Constraints}

This section shows that the acceleration constraint of the noise shaping filter is achieved in simulation, where the allowable acceleration variations have a specified cutoff frequency below $\omega_{\alpha c}=1.26 \mathrm{rad} / \mathrm{sec}$, and the variations is less than or equal to $\alpha_{\max }=1.2 \mathrm{~m} / \mathrm{s}^{2}$ for passenger comfort [19]. To comply with the cutoff frequency constraint, the $-3 \mathrm{~dB}$ cutoff frequency was set to $\omega_{\alpha c}=1.26 \mathrm{rad} / \mathrm{sec}$ using the method described in Section III-A, where $\rho$ was set to 0.5 , which corresponds to a $-3 \mathrm{~dB}$ attenuation at the cutoff frequency, as seen in Figure 8. Figure 9 (a) verifies that the acceleration variation does not extend past $1.2 \mathrm{~m} / \mathrm{s}^{2}$.

\section{B. Results for Position Variation Constraint}

This section shows that the position constraints of the noise shaping filter are achieved in simulation. The maximum allowable position from the center of the road depends on the width of the vehicle and the width of the lane, and it is assumed that the vehicle safely stays inside the lane if the center of the back axle is within $\pm 0.88 \mathrm{~m}$ of the center of the lane. Therefore, the maximum allowable lateral variation is $\delta_{\max }=0.88 \mathrm{~m}$. The results of the vehicle's trajectory after 250 simulations with $K=0.38$ can be seen in Figure 9 (b).

Figure 9 (b) shows that none of the trajectories are beyond the $\delta_{\max }$ boundary, therefore the system complies with the position constraints.

\section{Results for Delivering a Desired Average Charge within a Probability Interval}

This section shows that an average charge can be attained within a specified probability interval. For this test, the specified average charge was 40 kilowatts with a confidence interval of 0.97 . To calculate the corresponding $K$ value that achieves the desired charge, (35) is used which corresponds to the dashed line in Figure 10 (a). Figure 10 (a) depicts a $z$ value of 1.88 , which corresponds to a 0.97 lower tail integral of the Cumulative Distribution Function. Using the coefficients from (31) and (33), the corresponding noise shaping filter gain $K$ value is 0.38 . Figure 10 (b) shows the results of the 250 simulations, and that the probability of receiving an expected charge of 40 kilowatts or more was $96.8 \%$ as expected. The results from this section show that variation can be added to a vehicle's optimal charging trajectory while ensuring a desired average charge in addition to position and acceleration charging constraints.

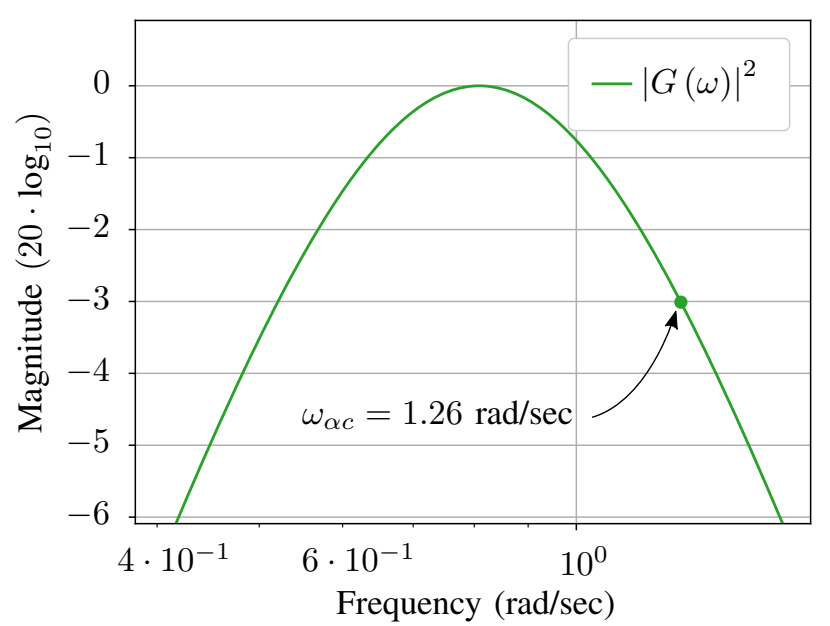

Figure 8: Plot verifying that the $-3 \mathrm{~dB}$ upper cutoff frequency of acceleration variation is less than or equal to $1.26 \mathrm{rad} / \mathrm{sec}$.

\section{Simulation Results for Desired Change in SOC}

This section tests that the path planner adequately sizes the output of the noise shaping filter based on a desired change in SOC in watt-hours. The vehicle profile from Table I is utilized with the maximum lateral misalignment $\delta_{\max }$ being extended to 1.76 meters. The value of $\delta_{\max }$ was increased to show a more dynamic contrast between noise variations based on differing road grades. The initial battery charge was set to 15 kilowatt-hours with a desired $\Delta Q$ equal to 300 watthours, which corresponds to a desired end charge of 15.3 kilowatt-hours. The simulated vehicle travels a 1900 meter track with two 400-meters charging regions, the WPT charging regions are centered at 500 meters and 1300 meters of the path respectively, and the grade of the path changes from $3 \%$ to $2 \%$ at 900 meters as depicted in Figure 11 (a).

The change in path grade between the two charging regions is used to test the ability of the path planner to adequately decrease the noise sizing to accommodate the reduction of average power consumption. The orange dots in Figure 11 (a) symbolize when the path planner calculates a new control variation sizing based on the average power usage of the previous 10 seconds, and the red dots symbolize when a maximum path variation is set after a charging region is complete.

A Monte Carlo simulation of 250 runs was completed for a dynamically charging vehicle, and the results can be seen in Figure 11 (b), which shows that the path planner achieves the desired end SOC without a priori knowledge of the path grade. At 200 meters, the first calculation for the desired average charge was set based on the $3 \%$ grade using the average power consumption over the previous 10 seconds. The calculation was intentionally made 10 seconds prior to the beginning of the charging station to allow the variance of the control variation to settle to steady-state before the beginning of the charging region. At 900 meters, the road grade was reduced to $2 \%$, which resulted in less power consumed by the battery than previously calculated. Therefore, when the desired charge was again computed at 700 meters, the path planner determined 


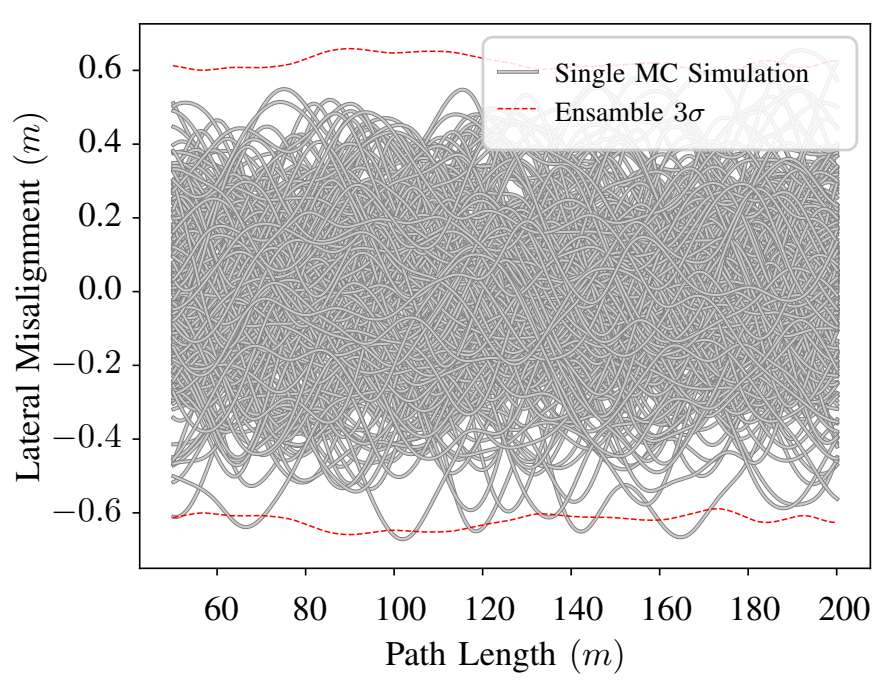

(a)

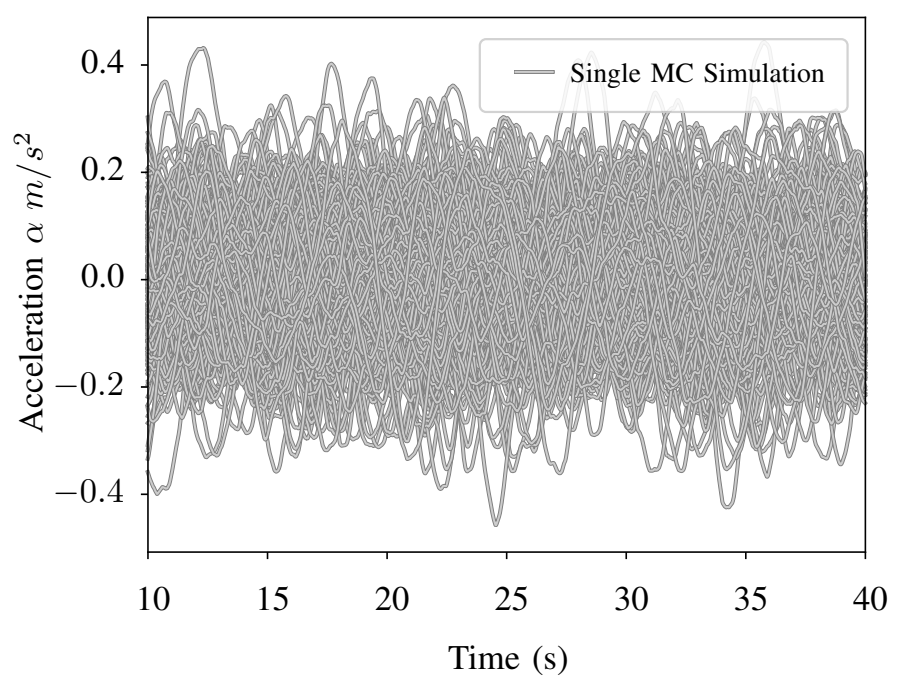

(b)

Figure 9: (a) Plot verifying that the position maximum constraint $\delta_{\max }$ is not violated by the noise variation. This figure demonstrates that all 250 Monte Carlo simulations are successfully within the $\delta_{\max }$ of $0.88 \mathrm{~m}$ for the case that $K=0.38$ by showing that the three standard deviation boundary is within the maximum position boundary. (b) Plot verifying that the acceleration maximum constraint of $\alpha_{\max }=1.2 \mathrm{~m} / \mathrm{s}^{2}$ is not violated for the noise variation for 250 Monte Carlo simulations when $K=0.38$.

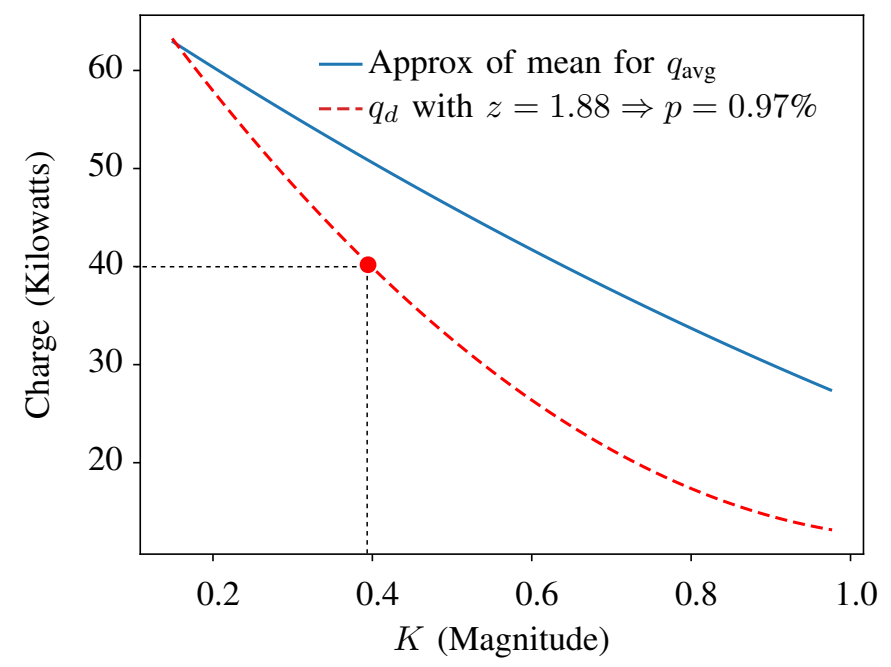

(a)

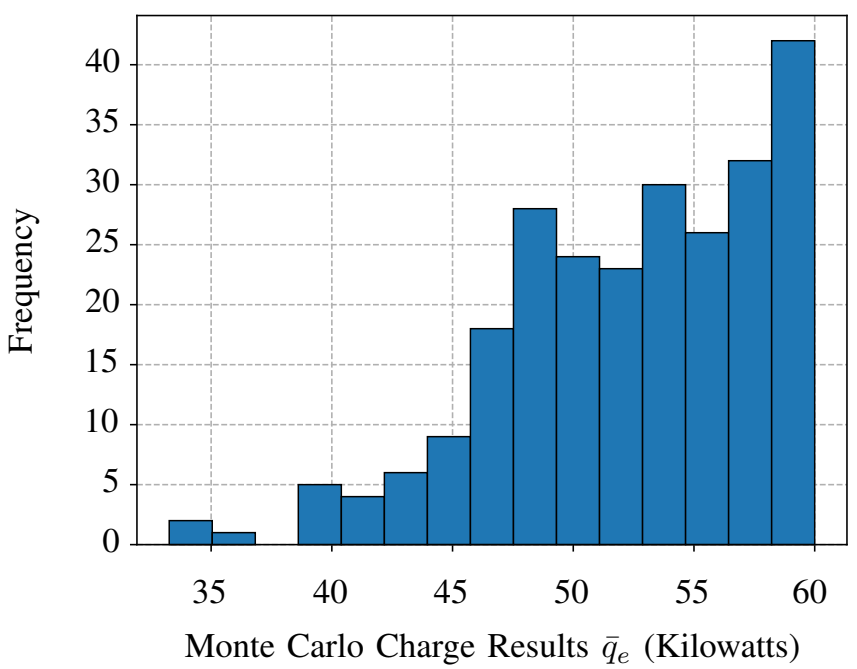

(b)

Figure 10: (a) Finding a $K$ value that yields an expected charge $q_{d}=40 \mathrm{~kW}$ with a probability of $p=.97$. The red-dotted line represents 1.88 standard deviations from average charge, which corresponds to a $97 \%$ upper tail probability interval. (b) Histogram plot testing that $97 \%$ of simulations had an expected charge above 40 kilowatts. This figure demonstrates that of the 250 simulations, $96.8 \%$ of the simulations were above 40 kilowatts.

that less charge was needed to arrive at the destination than previously solved for, so the noise sizing was increased.

The desired final battery state was $15.3 \mathrm{kWh}$ with $97 \%$ probability and the test resulted in $95.6 \%$, as seen in the histogram of Figure 12. These results verify that the path planner correctly sizes the output of the noise shaping filter for a specified change in the state of charge.

Figure 13 illustrates the effect of the proposed algorithm on vehicle-induced road wear as quantified by the maximum normalized strain described in section II-B. The blue curve represents the road wear associated with a naive controller that seeks to maximize charge by maintaining the vehicle within $20 \mathrm{~cm}$ of the center of the lane. The red curve represents the benefit of the proposed algorithm, which reduces the road wear throughout the path. The amount of reduction varies with the road grade and desired state of charge, achieving a factor 0.65 reduction in the first charging region and 0.5 in the second charging region where the road grade is lower. 


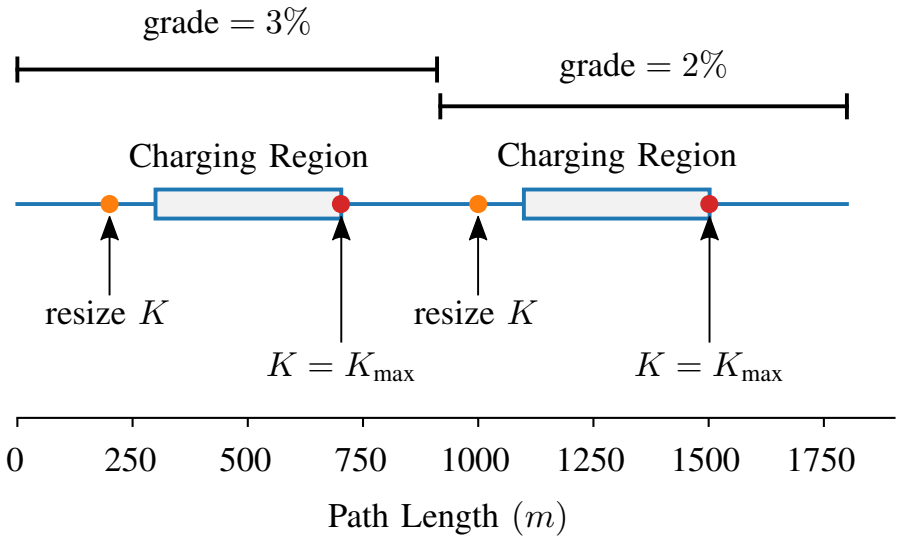

(a)

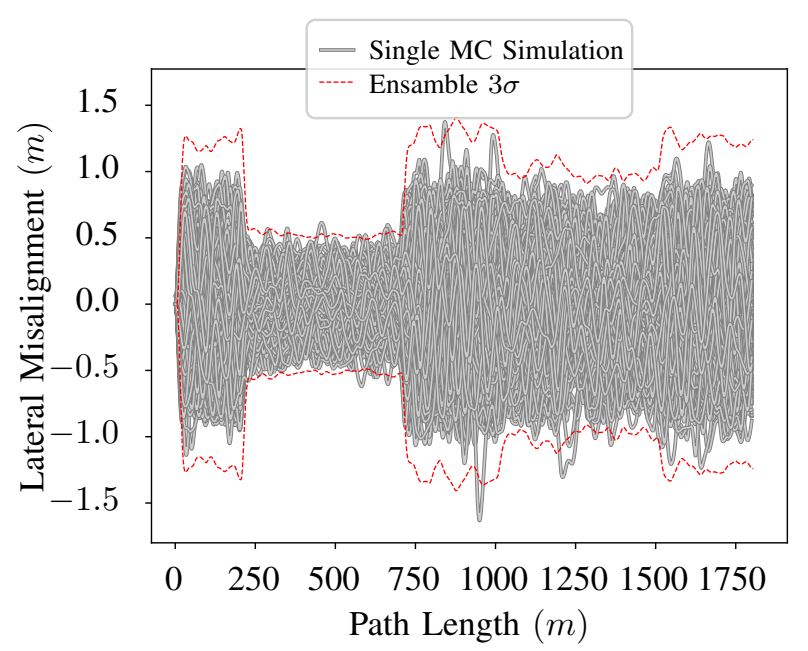

(b)

Figure 11: (a) Representation of the straight simulation test track used to test that the noise shaping filter can be sized to obtain a desired charge while injecting variation into the vehicle path. (b) Lateral misalignment results of 250 Monte Carlo simulations for a dynamically charging vehicle on the simulation test track.

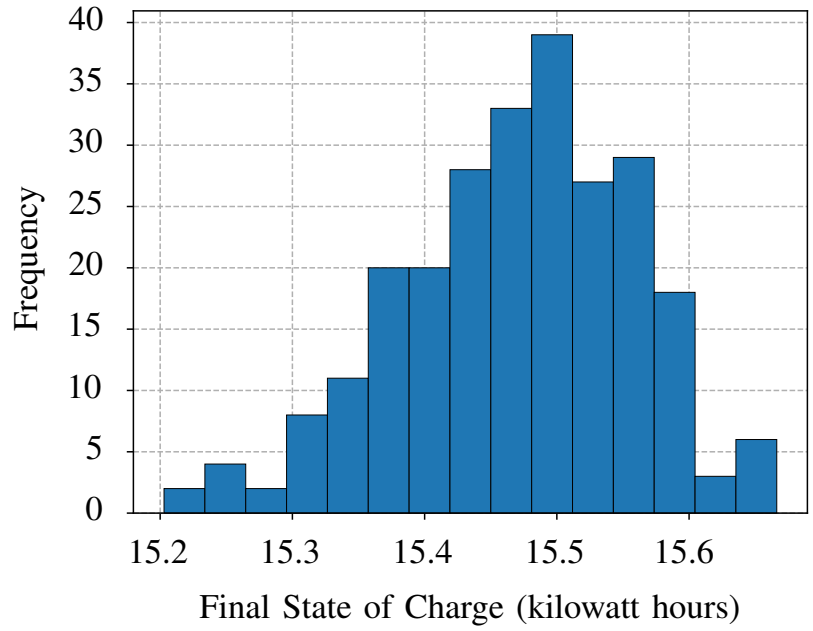

Figure 12: Histogram showing the battery SOC at the vehicle's destination. Of the 250 simulations, $96 \%$ were expected to be above 15300 watt-hours, and the result was $95.6 \%$.

\section{CONCLUSION}

In this paper, an algorithm is presented which reduces road wear by injecting filtered noise into the path of an autonomous dynamic charging vehicle. The algorithm ensures the vehicle achieves a specified average charge rate within a specified confidence interval while complying with systemspecific passenger comfort constraints. The algorithm is further developed to attain a final state of charge (SOC) within a specified probability, given a vehicle discharge model.

The performance of the algorithm was demonstrated via Monte Carlo analysis, and the designed noise shaping filter was shown to achieve the desired outcomes for a $1.8 \mathrm{~km}$ section of road with varying grades and discrete charging sections. It was shown that a specified SOC of $15.3 \mathrm{~kW}$ hours was achieved in $95.6 \%$ of the Monte Carlo simulations with a specified confidence interval of $96 \%$ while reducing

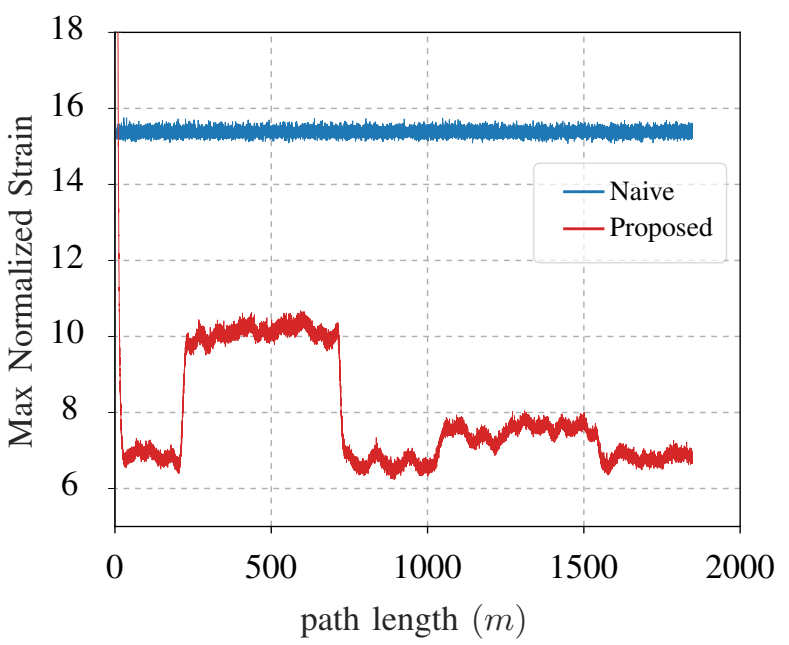

Figure 13: Normalized plastic road strain when $N=10,000$ for the test track.

the maximum normalized strain on the road by a factor between 0.5 and 0.65 on the charging regions. Additionally, the simulation results show that neither the maximum acceleration change of $1.2 \mathrm{~m} / \mathrm{s}$ nor the maximum acceleration frequency of $0.2 \mathrm{~Hz}$ was violated, and that the vehicle stayed within the maximum deviation from the center of the lane. This paper, therefore, shows that the proposed algorithm reduces normalized plastic strain on the road during wireless charging when compared to the naïve approach of always maximizing charge while ensuring an end SOC and maintaining passenger comfort constraints.

In addition to increasing the road-life around wireless charging regions, immediate applications of this research extend to reducing the road wear caused by the repeated history of heavy autonomous mining equipment, which causes significantly more road damage as a consequence of their weight and repeated use [33]. 


\section{APPENDIX A \\ NoISE ShAPING Filter Form}

The band-pass filter $G(s)$ proposed in Section II-A is formed by multiplying a second-order low-pass filter and second-order a high-pass filter. A second-order low-pass filter $G_{l}(s)$ and second-order high-pass filter $G_{h}(s)$ have transfer functions of

$$
\begin{aligned}
G_{l}(s) & =\frac{\omega_{l}^{2}}{s^{2}+2 \zeta \omega_{l} s+\omega_{l}^{2}} \\
G_{h}(s) & =\frac{s^{2}}{s^{2}+2 \zeta \omega_{h} s+\omega_{h}^{2}}
\end{aligned}
$$

with corresponding system magnitudes in the frequency domain of

$$
\begin{aligned}
\left|G_{l}(j \omega)\right|^{2} & =\frac{\omega_{l}^{4}}{\omega^{4}+2 \omega_{l}^{2}\left(2 \zeta^{2}-1\right) \omega^{2}+\omega_{l}^{4}} \\
\left|G_{h}(j \omega)\right|^{2} & =\frac{\omega^{4}}{\omega^{4}+2 \omega_{h}^{2}\left(2 \zeta^{2}-1\right) \omega^{2}+\omega_{h}^{4}} .
\end{aligned}
$$

Therefore, the magnitude squared of a band-pass filter scaled by $K$ is

$$
\begin{aligned}
|G(j \omega)|^{2} & =|K|^{2} \cdot\left|G_{l}(j \omega)\right|^{2} \cdot\left|G_{h}(j \omega)\right|^{2} \\
|G(j \omega)|^{2} & =K^{2} \cdot \frac{\omega^{4}}{\omega^{4}+2 \omega_{h}^{2}\left(2 \zeta^{2}-1\right) \omega^{2}+\omega_{h}^{4}} \cdot \cdots \\
& \frac{\omega^{4}}{\omega^{4}+2 \omega_{h}^{2}\left(2 \zeta^{2}-1\right) \omega^{2}+\omega_{h}^{4}} .
\end{aligned}
$$

Let $\zeta=\frac{1}{\sqrt{2}}$ then,

$$
|G(j \omega)|^{2}=\frac{K^{2} \omega_{l}^{4} \omega^{4}}{\left(\omega^{4}+\omega_{l}^{4}\right)\left(\omega^{4}+\omega_{h}^{4}\right)} .
$$

Because variation in position is the double integral of acceleration, the transfer function for position becomes

$$
H(s)=G(s) \frac{1}{s^{2}}
$$

and the magnitude squared of the frequency domain assuming is $\zeta=\frac{1}{\sqrt{2}}$ becomes

$$
|H(j \omega)|^{2}=\frac{K^{2} \omega_{l}^{4}}{\left(\omega^{4}+\omega_{l}^{4}\right)\left(\omega^{4}+\omega_{h}^{4}\right)} .
$$

\section{APPENDIX B}

\section{Setting the Magnitude of The Band-Pass Filter at THE CUTOFF FREQUENCY}

This derivation shows how to select values for $\omega_{l}$ and $\omega_{h}$ such that the system magnitude at the cutoff frequency $\omega_{\alpha c}$ for the band-pass filter is equal to $\rho$. First, recall that the band-pass filter in the frequency domain $|G(j \omega)|$ is composed of a second-order low-pass filter $\left|G_{\mathrm{lp}}(j \omega)\right|$ multiplied by a second-order high-pass filter $\left|G_{\mathrm{hp}}(j \omega)\right|$. As a result, the system $|G(j \omega)|$ will always have a maximum magnitude less than one. However, for this application it is desirable to scale $|G(j \omega)|$ such that the maximum value of the band-pass filter is one, and the cutoff frequency can be calculated in terms of unity pass-band.
To find the maximum value of $|G(j \omega)|$, the geometric properties of the band-pass filter can be leveraged. The frequency that corresponds to the maximum value of $|G(j \omega)|$ occurs at the logarithmic midpoint $\omega_{m}$ which is centered between $\omega_{l}$ and $\omega_{h}$ as depicted in Figure 14.

The midpoint $\omega_{m}$ between two logarithmic points is

$$
\begin{aligned}
& \omega_{m}=10^{\frac{\log _{10}\left(\omega_{l}\right)+\log _{10}\left(\omega_{h}\right)}{2}} \\
& \omega_{m}=\sqrt{\omega_{l} \omega_{h}},
\end{aligned}
$$

which implies that the maximum value of $|G(\omega)|_{\max }=$ $\left|G\left(\omega_{m}\right)\right|=\frac{\omega_{l}^{2}}{\omega_{h}^{2}+\omega_{l}^{2}} \leq 1$. Therefore the scale factor $K_{s}$ that sets the maximum value of the band-pass filter to one is

$$
K_{s}=\left|G\left(\omega_{m}\right)\right|^{-1}=\frac{\omega_{h}^{2}+\omega_{l}^{2}}{\omega_{l}^{2}} .
$$

Equation (62) can then be written in terms of the design parameter $\kappa$ where $\omega_{h}=\kappa \omega_{l}$ as presented in Section III-A. With the band-pass filter scaled to one, the value of $\omega_{l}$ that fixes the magnitude of the cutoff frequency to $\rho$ can be solved for using the following equation

$$
\begin{aligned}
|G(j \omega)|=\rho & =\frac{K_{s} \omega_{l}^{2} \omega^{2}}{\sqrt{\left(\omega^{4}+\omega_{l}^{4}\right)\left(\omega^{4}+\omega_{h}^{4}\right)}} \\
\rho & =\frac{\left(\frac{\left(\kappa \omega_{l}\right)^{2}+\omega_{l}^{2}}{\omega_{l}^{2}}\right) \omega_{l}^{2} \omega_{\alpha c}^{2}}{\sqrt{\left(\omega_{\alpha c}^{4}+\omega_{l}^{4}\right)\left(\omega_{\alpha c}^{4}+\left(\kappa \omega_{l}\right)^{4}\right)}} \\
-\frac{\omega_{\alpha c}^{8}}{\kappa^{4}} & =\omega_{l}^{8}+\omega_{\alpha c}^{4}\left(1+\frac{1}{\kappa^{4}}-\frac{\left(\kappa^{2}+1\right)^{2}}{\kappa^{4} \rho^{2}}\right) \omega_{l}^{4}
\end{aligned}
$$

Let $\Omega=\omega_{\alpha c}^{4}\left(1+\frac{1}{\kappa^{4}}-\frac{\left(\kappa^{2}+1\right)^{2}}{\kappa^{4} \rho^{2}}\right)$, then

$$
\begin{aligned}
-\frac{\omega_{\alpha c}^{8}}{\kappa^{4}} & =\omega_{l}^{8}+\Omega \omega_{l}^{4} \\
\omega_{l} & =\left(-\sqrt{-\frac{\omega_{\alpha c}^{8}}{\kappa^{4}}+\frac{1}{4} \Omega^{2}}-\frac{1}{2} \Omega\right)^{1 / 4}
\end{aligned}
$$

and $\omega_{h}=\kappa \omega_{l}$.

\section{REFERENCES}

[1] US Department of Energy , “ U.S. Plug-in Electric Vehicle Sales by Model ," 2017. [Online]. Available: https://afdc.energy.gov/data/10567

[2] J. Guzowski. (2017) Wave announces wireless charging with avta at lancaster city park and palmdale transportation center. [Online]. Available: "https://busride.com/ wave-announces- wireless-charging-antelope-valley/"

[3] Y. J. Jang, S. Jeong, and M. Seok Lee, "Initial energy logistics cost analysis for stationary, quasi-dynamic, and dynamic wireless charging public transportation systems," Energies, vol. 9, p. 483, 062016.

[4] Z. Bi, T. Kan, C. C. Mi, Y. Zhang, Z. Zhao, and G. A. Keoleian, "A review of wireless power transfer for electric vehicles: Prospects to enhance sustainable mobility," Applied Energy, vol. 179, pp. $413-$ 425, 2016. [Online]. Available: http://www.sciencedirect.com/science/ article/pii/S0306261916309448

[5] K. Hwang, J. Park, D. Kim, H. H. Park, J. H. Kwon, S. I. Kwak, and S. Ahn, " Autonomous Coil Alignment System Using Fuzzy Steering Control for Electric Vehicles with Dynamic Wireless Charging " Mathematical Problems in Engineering, vol. 2015, pp. 1-14, dec 2015.

[6] J. Kim , J. Kim, S. Kong, H. Kim, I. Suh, N. P. Suh, D. Cho , J. Kim , and S. Ahn, "Coil design and shielding methods for a magnetic resonant wireless power transfer system," Proceedings of the IEEE, vol. 101, no. 6, pp. 1332-1342, June 2013. 


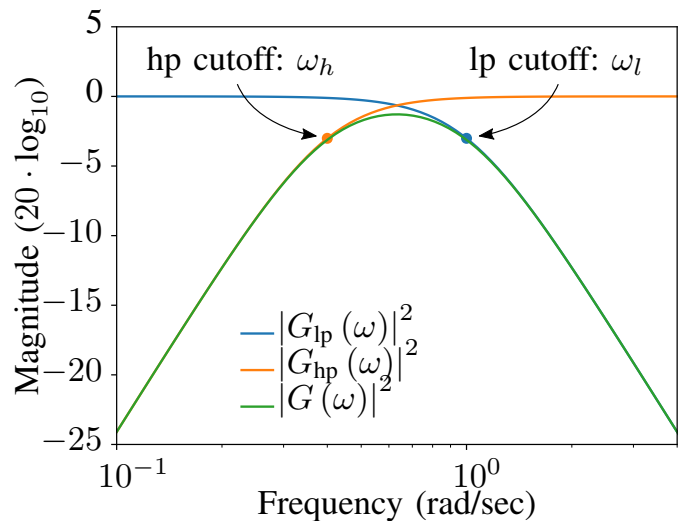

Figure 14: Depiction that the logarithmic midpoint between a low-pass filter cutoff frequency $\omega_{l}$ and a high-pass filter cutoff frequency $\omega_{h}$ constitutes the frequency of the maximum value for the band-pass filter, $|G(\omega)|^{2}$.

[7] J. Shin, S. Shin, Y. Kim, S. Ahn, S. Lee, G. Jung, S. Jeon , and D. Cho , "Design and implementation of shaped magneticresonance-based wireless power transfer system for roadway-powered moving electric vehicles," IEEE Transactions on Industrial Electronics, vol. 61, no. 3, pp. 1179-1192, March 2014.

[8] J. P. W. Chow , N. Chen, H. S. H. Chung, and L. L. H. Chan , "An investigation into the use of orthogonal winding in loosely coupled link for improving power transfer efficiency under coil misalignment," IEEE Transactions on Power Electronics, vol. 30, no. 10, pp. 5632-5649, Oct 2015.

[9] Y. Gao, K. Blair Farley, and Z. Tse, "A uniform voltage gain control for alignment robustness in wireless ev charging," Energies, vol. 8, pp. 8355-8370, 082015.

[10] S. Y. Choi , S. Y. Jeong, E. S. Lee, B. W. Gu, S. W. Lee, and C. T. Rim, "Generalized models on self-decoupled dual pick-up coils for large lateral tolerance," IEEE Transactions on Power Electronics, vol. 30, no. 11, pp. 6434-6445, Nov 2015.

[11] S. F. Said and H. Hakim, "Effects of transversal distribution of heavy vehicles on rut formation in bituminous layers," Swedish National Road and Transport Research Institute, Tech. Rep., 2018.

[12] L. Chu, G. Ping Ong, T. F. Fwa, and G. P. Ong, "Evaluating Hydroplaning Potential of Rutted Highway Pavements Disruption Modeling for Public Transportation Systems View project Sustainable Transportation Planning and Policy in Malaysia View project Evaluating Hydroplaning Potential of Rutted Highway Pa," Journal of the Eastern Asia Society for Transportation Studies, vol. 11, 2015. [Online]. Available: https://www.researchgate.net/publication/317868902

[13] J. N. Ivan, N. Ravishanker, E. Jackson, B. Aronov, and S. Guo, "A Statistical Analysis of the Effect of Wet-Pavement Friction on Highway Traffic Safety,” Journal of Transportation Safety \& Security, 2012.

[14] Hjort Mattias, Mattias Haraldsson, and Jansen Jan, "Road Wear from Heavy Vehicles - an overview," NVF committee Vehicles and Transports, Tech. Rep., 2008.

[15] H. L. Von Quintus, J. Mallela, L. Titus-Glover, and Asphalt Institute, Calibration factors for polymer-modified asphalts using $M-E$ based design methods. Asphalt Institute, 2008, OCLC: 422752841.

[16] Y. S. Byun and Y. C. Kim, "Localization based on magnetic markers for an all-wheel steering vehicle," Sensors (Switzerland), 2016.

[17] M. Cornick, J. Koechling, B. Stanley, and B. Zhang, "Localizing Ground Penetrating RADAR: A Step Towards Robust Autonomous Ground Vehicle Localization," Ph.D. dissertation, MIT Lincoln Laboratory, 2016.

[18] K. Hwang, J. Cho, D. Kim, J. Park, J. H. Kwon, S. I. Kwak, H. H. Park, and S. Ahn, "An autonomous coil alignment system for the dynamic wireless charging of electric vehicles to minimize lateral misalignment , Energies, vol. 10, no. 3, 2017

[19] J. Eriksson and L. Svensson, "Tuning for Ride Quality in Autonomous Vehicle Application to Linear Quadratic Path Planning Algorithm," Ph.D. dissertation, Uppsala Universitet, 2015. [Online]. Available: https://www.diva-portal.org/smash/get/diva2:839140/FULLTEXT01.pdf

[20] J. F. Golding, "A motion sickness maximum around the $0.2 \mathrm{~Hz}$ frequency range of horizontal translational oscillation," Aviation
Space and Environmental Medicine, 2001. [Online]. Available: https://www.researchgate.net/publication/12056741

[21] I. O. for Standardization, "Mechanical vibration and shock - evaluation of human exposure to whole-body vibration," International Organization for Standardization, Geneva, CH, Standard, Jul. 1997.

[22] S. K. Sharma and A. Kumar, "Ride comfort of a higher speed rail vehicle using a magnetorheological suspension system," Proceedings of the Institution of Mechanical Engineers, Part K: Journal of Multi-body Dynamics, vol. 232, no. 1, pp. 32-48, 2018. [Online]. Available: https://doi.org/10.1177/1464419317706873

[23] R. C. Sharma and S. K. Sharma, "Sensitivity analysis of three-wheel vehicle's suspension parameters influencing ride behavior," Noise \& Vibration Worldwide, vol. 49, no. 7-8, pp. 272-280, Jul. 2018. [Online]. Available: http://journals.sagepub.com/doi/10.1177/0957456518796846

[24] S. Palli, R. Koona, S. Sharma, and R. Sharma, "A Review on Dynamic Analysis of Rail Vehicle Coach," International Journal of Vehicle Structures and Systems, vol. 10, no. 3, Aug. 2018. [Online]. Available: http://maftree.org/eja/index.php/ijvss/article/view/1077

[25] S. K. Sharma, U. Saini, and A. Kumar, "Semi-active Control to Reduce Lateral Vibration of Passenger Rail Vehicle Using Disturbance Rejection and Continuous State Damper Controllers," Journal of Vibration Engineering \& Technologies, vol. 7, no. 2, pp. 117129, Apr. 2019. [Online]. Available: http://link.springer.com/10.1007/ s42417-019-00088-2

[26] S. K. Sharma and R. C. Sharma, "Simulation of Quarter-Car Model with Magnetorheological Dampers for Ride Quality Improvement," International Journal of Vehicle Structures and Systems, vol. 10, no. 3, Aug. 2018. [Online]. Available: http://maftree.org/eja/index.php/ijvss/ article/view/1070

[27] J. Villagra, V. Milanés, J. Pérez, and J. Godoy, "Smooth path and speed planning for an automated public transport vehicle," Robotics and Autonomous Systems, 2012.

[28] P. Maybeck, Stochastic Models, Estimation, and Control, ser. Mathematics in Science and Engineering. Elsevier Science, 1982. [Online]. Available: https://books.google.com/books/about/Stochastic_ Models_Estimation_and_Control.html?id=FkkZBqQG36gC

[29] R. V. Siddharthan, M. Nasimifar, X. Tan, and E. Y. Hajj, "Investigation of impact of wheel wander on pavement performance," Road Materials and Pavement Design, vol. 18, no. 2, pp. 390-407, 2017. [Online]. Available: https://www.tandfonline.com/doi/full/10.1080/14680629.2016.1162730

[30] K. Young, C. Wang, L. Y. Wang, and K. Strunz, "Electric vehicle battery technologies," in Electric Vehicle Integration into Modern Power Networks, 2013

[31] W.-Y. Chang, " The State of Charge Estimating Methods for Battery: A Review ," ISRN Applied Mathematics, vol. 2013, pp. 1-7, 2013. [Online]. Available: https://www.hindawi.com/archive/2013/953792/

[32] M. A. Hannan, M. S. Lipu, A. Hussain, and A. Mohamed, " A review of lithium-ion battery state of charge estimation and management system in electric vehicle applications: Challenges and recommendations," pp. 834-854, 2017.

[33] D. Tannant and B. Regensburg, "Guidelines for mine haul road design," 2001. [Online]. Available: https://open.library.ubc.ca/ collections/facultyresearchandpublications/52383/items/1.0102562

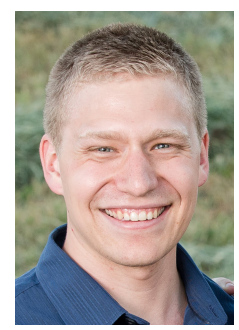

Clint Ferrin graduated from Utah State University in 2019 with his Maters's Degree in Electrical Engineering with an emphasis in Control Theory for Mobile Robotics. Clint is currently working for the United States Air Force to produce high fidelity simulations, and he plans to continue his learning and research.

His research interests include mobile robotics, nonholonomic constrained motion planning, and nonlinear, optimal control.

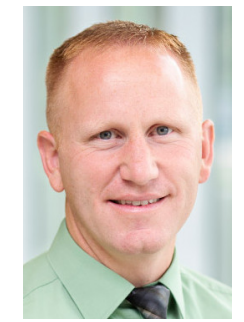

Randall Christensen is an assistant professor in the Department of Electrical and Computer Engineering at Utah State University, specializing in guidance, navigation, and control of autonomous vehicles. He received his Ph.D. in Mechanical and Aerospace engineering in 2013. Dr. Christensen joined the USU faculty after 12 years in the aerospace industry working on a wide range of projects, including turreted weapons, SmallSats, and missile GNC systems. His research focuses on closed-loop linear covariance analysis, GPS-denied navigation, and terrain-relative navigation for space missions. 